\title{
Micropatterning of Bioactive Glass Nanoparticles on Chitosan Membranes for Spatial Controlled Biomineralization
}

\author{
Gisela M. Luz, ${ }^{\dagger, \ddagger}$ Luciano Boesel, ${ }^{\S}$ Aránzazu del Campo, ${ }^{\S}$ and João F. Mano ${ }^{*}, \dagger$ \\ ${ }^{\dagger} 3$ B’s Research Group-Biomaterials, Biodegradables and Biomimetics, University of Minho, Headquarters of the European Institute of \\ Excellence on Tissue Engineering and Regenerative Medicine, AvePark, 4806-909 Caldas das Taipas, Portugal \\ ${ }^{*}$ ICVS/3B's-PT Government Associated Laboratory, Braga/Guimarães, Portugal \\ ${ }^{\S}$ Max Planck Institute for Polymer Research, Ackermannweg 10, 55128 Mainz, Germany
}

ABSTRACT: Bioactive glass nanoparticles (BG-NPs) capable of inducing apatite precipitation upon immersion in simulated body fluid (SBF) were patterned on free-standing chitosan membranes by microcontact printing using a poly(dimethylsiloxane) (PDMS) stamp inked in a BG-NPs pad. Formation of the patterns was characterized by scanning electron microscopy (SEM). Mineralization of the bioactive glass patterns was induced in vitro by soaking the samples in SBF over different time points up to 7 days. The confined apatite deposition in the patterned regions with diameters of $50 \mu \mathrm{m}$ was confirmed by Fourier-

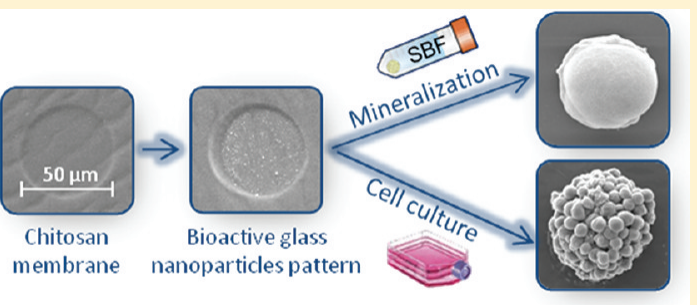
transformed infrared spectroscopy (FTIR), energy-dispersive X-ray (EDX) analysis, and SEM. In vitro tests confirmed the preferential attachment and proliferation of L929 cells to the areas printed with BG-NPs of the membranes. This approach permits one to spatially control the properties of biomaterials at the microlevel and could be potentially used in guided tissue regeneration for skin, vascular, articular, and bone tissue engineering and in cellular cocultures or to develop substrates able to confine cells in regions with controlled geometry at the cell's length scale.

\section{INTRODUCTION}

In recent years, the biomaterials field has witnessed the rise of a third generation of materials able to stimulate specific cellular responses. ${ }^{1,2}$ Exposed to the right surface chemistry and topography, cells can adhere, proliferate, and differentiate. ${ }^{3-6}$ Tailoring of surfaces morphology can be done along the $Z$ direction by promoting roughness (including hierarchical features) or patterning regular motifs. Chemically, the surface can be modified, for example, by layer-by-layer constructs, sequential attachment of chemical/biochemical elements, and also grafting smart macromolecules. Changes along the $X-Y$ direction enclose creation of geometrical domains, regular stripes, or gradients. ${ }^{3}$ It has been demonstrated that the size of cell-binding domains may have a direct influence in cellular behavior, including differentiation, ${ }^{4}$ and anisotropic patterns could direct preferential cell alignment. ${ }^{7}$

Different methods can be applied to engineer culture substrates for guiding cellular responses with spatial control. Microcontact printing $(\mu \mathrm{CP})$ of biologically relevant ligands ${ }^{8-10}$ using a soft poly(dimethylsiloxane) PDMS stamp is the most common technique to generate specific patterns with different and well-defined chemistries. Patterns of proteins, molecules, polymers, nanoparticles, self-assembled monolayers, colloids, and metals have been reported. ${ }^{11-14}$ Application of this patterning technology to cell culture engineering provides new tools for spatially controlled tissue engineering. The flexibility of sizes and shapes of the patterns have allowed generation of patterned cell cocultures that facilitate cell proliferation and differentiation and also engineering of tissue constructs. ${ }^{8,15}$

Bioactive glass has been demonstrated to have a beneficial effect in bone regeneration, skin, articular regeneration, and angiogenesis applications as it binds to both bone and soft tissue. ${ }^{16}$ Bioactive glass has been mainly applied in orthopedic and dental areas, since it promotes deposition of apatite under physiological conditions. ${ }^{1,17,18}$ A few works have reported the fabrication of substrates with spatial control of biomineralization. ${ }^{19,20} \mu \mathrm{CP}$ has not been used for this purpose, though it presents significant advantages over the reported methods. (i) It is based on a very simple procedure, easily adjustable to different substrates that do not require inherent bioactivity. (ii) No external stimuli are required to trigger the beginning of mineralization, ${ }^{20}$ and (iii) no organic solvents are used in the process which would not be an appropriate choice when working with polymers. ${ }^{19}$ In addition, the major advantage of applying BG-NPs patterns for tissue culture applications is the fact that bioactive glasses are gene-activation materials capable of inducing cellular differentiation. ${ }^{21}$ Therefore, development of BG-NPs patterns will provide a versatile tool for biological studies, since they are inexpensive and also more resistant to temperature, storing, and sterilization procedures than the molecular components often used in biology research. Moreover, this system would also be very useful in bioactive

Received: November 28, 2011

Revised: April 2, 2012 
glass-related studies, since their formulation, sizes, and geometry influence their bioactivity and cellular interactions.

This paper describes a methodology to obtain micropatterned bioactive glass nanoparticles (BG-NPs) on chitosan membranes by $\mu \mathrm{CP}$. Ionic dissolution of $\mathrm{Si}, \mathrm{Ca}$, and $\mathrm{P}$ gives rise to both intracellular and extracellular responses at the interface of the glass with its cellular environment which stimulate cells to grow and differentiate, influencing as well their biomechanical properties. ${ }^{1}$ Instead of producing homogeneous nanocomposites with BG-NPs distributed in all their volume we opted for another strategy in which the inorganic fraction is deposited over the polymeric membrane. Nanocomposites produced by combining BG-NPs and polymers ${ }^{22}$ are inspired by mineralized structures found in Nature. ${ }^{23}$

Moreover, this work aims to achieve spatial control of biomineralization using $\mu \mathrm{CP}$ of BG-NPs to generate mineralizable patterns. $\mu \mathrm{CP}$ allows precise control of nanoparticles density at the surface, thereby controlling ionic release from the BG-NPs and avoiding inefficient particles concentration toward mineralization or excessive release of ions that locally change the $\mathrm{pH}$ of the environment to levels lethal to the cells. This is important when working at the nanolevel, since unlike bulk materials the high specific surface area of BG-NPs increases their degradability. ${ }^{12,24-26}$ In addition, nanosized particles can cause different biological responses in comparison with the ones obtained for larger particles with the same chemical composition. One example is the enhancement of cell attachment due to the nanoparticles higher surface reactivity, which increases protein adsorption. ${ }^{27,28}$

Combining both physical and chemical strategies to control cell attachment and apatite deposition we expect to create an innovative, tissue engineering platform that could have potential applications in different regeneration fields.

\section{EXPERIMENTAL SECTION}

BG-NPs Preparation. The procedure to produce BG-NPs with composition $\mathrm{SiO}_{2}: \mathrm{CaO}: \mathrm{P}_{2} \mathrm{O}_{5}(\mathrm{~mol} \%)=55: 40: 5$ was based on a previously reported protocol. ${ }^{29}$ Tetraethyl orthosilicate (TEOS, 99.90\% pure), diammonium hydrogen phosphate, calcium nitrate tetrahydrate (99\%), absolute ethanol, citric acid monohydrate (99\%), and ammonium hydroxide were purchased from Sigma-Aldrich. The mixture of precursor's solutions $(7.639 \mathrm{~g}$ of calcium nitrate in $120 \mathrm{~mL}$ of distilled water, $9.167 \mathrm{~g}$ of TEOS in $60 \mathrm{~mL}$ of ethanol and $30 \mathrm{~mL}$ of citric acid $10 \%(\mathrm{w} / \mathrm{v})$ ) was added drop-by-drop to an aqueous solution containing the phosphorus precursor (1.078 $\mathrm{g}$ of diammonium hydrogen phosphate) in $1500 \mathrm{~mL}$ of distilled water. In the case of the binary system, no phosphorus precursor was used in order to achieve the composition $\mathrm{SiO}_{2}: \mathrm{CaO}(\mathrm{mol} \%)=70: 30$.The $\mathrm{pH}$ was maintained at 11.5 with ammonium hydroxide addition. The precipitate obtained was stirred for $48 \mathrm{~h}$, and then a resting period of $24 \mathrm{~h}$ followed. The precipitate was washed three times with distilled water. A $200 \mathrm{~mL}$ amount of an aqueous solution of poly(ethylene glycol) $2 \%(\mathrm{w} / \mathrm{v})$ with $M_{\mathrm{w}}=20000$ was added to the precipitate, and then freeze drying followed. Finally, the BG nanoparticles were calcined at $700{ }^{\circ} \mathrm{C}$ for $3 \mathrm{~h}$ in order to achieve optimal conditions for bioactivity. ${ }^{30}$

PDMS Stamps Preparation. A master containing three different patterned fields was used. The geometries were $50 \mu \mathrm{m}$ diameter cylindrical pillars arranged in a square pattern with $50 \mu \mathrm{m}$ spacing, 50 $\mu \mathrm{m}$ diameter cylindical pillars arranged in a hexagonal pattern with 40 $\mu \mathrm{m}$ spacing, and ellipsoidal pillars (50 $\mu \mathrm{m}$ long axes and $30 \mu \mathrm{m}$ short axes) arranged in a hexagonal pattern with $40 \mu \mathrm{m}$ spacing. Each field was an $8 \mathrm{~mm}^{2}$ square. The master was fabricated by photolithography using SU-8 photolack. ${ }^{31}$ PDMS stamp was prepared by casting a 10:1 mixture of Sylgard 184 (Dow Corning) prepolymer and cross-linker. The mixture was poured over the master and cured at $90{ }^{\circ} \mathrm{C}$ for $3 \mathrm{~h}$ in a vacuum oven. After cooling, the PDMS was peeled off from the lithographic template and cut to suitable sizes.

Membranes Preparation. Membranes were obtained by solvent casting. Medium molecular weight chitosan with a degree of deacetylation of $79 \%$, purchased from Sigma-Aldrich, was dissolved in an aqueous acetic acid solution $2 \%(\mathrm{v} / \mathrm{v})$ to a concentration of $1 \%$ $(\mathrm{w} / \mathrm{v})$. An $80 \mathrm{~mL}$ amount of chitosan solution was casted onto $15 \mathrm{~mm}$ $\times 20 \mathrm{~mm}$ square Petri dishes and left for evaporation for 7 days. The dried membranes were neutralized by soaking in $\mathrm{NaOH} 0.1 \mathrm{M}$ for 10 min and washed with distilled water until water with a $\mathrm{pH}$ of 7 was reached. Membranes were left to dry at room temperature and clipped between 2 frames to obtain straight and smooth surfaces. They were then cut in order to obtain $7 \mathrm{~mm}$ diameter circles.

Patternings. A $549 \mu \mathrm{L}$ amount of BG-NPs dispersion with different nanoparticles concentrations in ethanol (between $0.003 \%$ and $0.05 \%)$ was poured on the surface of a glass slide $\left(9^{\circ}\right.$ inclination) in a small area of $1 \mathrm{~cm} \times 1.5 \mathrm{~cm}$ limited with a hollow rectangular piece fixed to the glass slide with dental wax. The dispersion was left to evaporate for $48 \mathrm{~h}$ inside a chamber saturated with ethanol. The lift-off was made by pressing the PDMS stamp against the dried membrane of BG-NPs that remained in the glass slide (donor substrate) for $10 \mathrm{~min}$ at $7 \mathrm{kPa}$ and room temperature. Before the "printing" step, the chitosan membrane was treated with a drop of acetic acid $(0.1 \mathrm{M})$ spread on its surface with a brush. $\mu \mathrm{CP}$ of the nanoparticles was achieved by pressing the BG-NPs-loaded stamp on the chitosan membrane for $30 \mathrm{~s}$ under $30 \mathrm{kPa}$ at room temperature. After removing the PDMS, the membrane was washed with ethanol and dried in a vacuum oven for $2 \mathrm{~h}$ at $40{ }^{\circ} \mathrm{C}$ followed by $24 \mathrm{~h}$ at $10^{-2}$ bar.

In Vitro Mineralization Study. In vitro mineralization tests were carried out by soaking patterned circular (diameter $7 \mathrm{~mm}$ ) chitosan membranes in $50 \mathrm{~mL}$ of simulated body fluid (SFB) solution for 0 (control), 1, 3, 5, and 7 days at $37{ }^{\circ} \mathrm{C}$. Upon removal from SBF, the membranes were rinsed with distilled water and left to dry. The SBF was prepared by dissolving $\mathrm{NaCl}, \mathrm{NaHCO}_{3}, \mathrm{KCl}, \mathrm{K}_{2} \mathrm{HPO}_{4} \cdot 3 \mathrm{H}_{2} \mathrm{O}$, $\mathrm{MgCl}_{2} \cdot 6 \mathrm{H}_{2} \mathrm{O}$, and $\mathrm{Na}_{2} \mathrm{SO}_{4}$ in distilled water and buffered with tris(hydroxymethyl)aminomethane buffer and $\mathrm{HCl}$ to reach a $\mathrm{pH}$ value of 7.4, following the protocol described by Kokubo and Takadama. ${ }^{32}$ All chemicals were purchased from Sigma-Aldrich.

Fourier-Transformed Infrared (FTIR) Spectroscopy Analysis. FTIR spectroscopy analysis of the formed hydroxyapatite on the chitosan membranes was carried out in an IR Prestige 21 Shimadzu Spectrometer. The hydroxyapatite powder was collected by scratching the surface of the membranes and pressed with $\mathrm{KBr}$ in a small disk. FTIR spectra were recorded from 400 to $4400 \mathrm{~cm}^{-1}$ with a resolution of $4 \mathrm{~cm}^{-1}$. Before measurements the powders were dried at $100{ }^{\circ} \mathrm{C}$ overnight.

Scanning Electron Microscopy (SEM) and Energy-Dispersive X-ray (EDX) Samples Preparation. A NanoSEM-FEI Nova 200 (FEG/SEM) scanning electron microscope was used to image the surface and morphology of the printed membranes. A conductive gold coating with $20 \mathrm{~nm}$ thickness was sputtered to the samples. A Pegasus $\mathrm{X} 4 \mathrm{M}$ instrument was used to perform the EDX experiments at low vacuum and without coating.

Cytotoxicity and Cell Attachment Tests. L929 mouse fibroblasts line (European collection of cell culture-ECACC, UK) was used to test the in vitro biocompatibility of the membranes. Chitosan membranes were previously sterilized by immersion in $70 \%$ (v/v) ethanol overnight and then washed twice with sterile phosphatebuffered saline (PBS). Cells were seeded in the samples $(n=3)$ at a density of 65000 cells $/ \mathrm{cm}^{2}$ nourished with Dulbecco's modified minimum essential medium (D-MEM) supplemented with $10 \%$ fetal bovine serum (FBS) and $1 \%$ antibiotic. Cultures were incubated at 37 ${ }^{\circ} \mathrm{C}$ for 1,3 , and 7 days. After each time point (1, 3, and 7 days of culture), MTS (3-(4,5-dimethylthiazol-2-yl)-5-(3-carboxymethoxyphenyl)-2-(4-sulfophenyl)-2 $\mathrm{H}$-tetrazolium) test was performed to determine the cytotoxicity of the membranes. The relative cellular viability (\%) was determined and compared with tissue culture polystyrene (TCPS) (positive control of cell viability). Latex was used as a negative control of cellular viability. For this assay, an MTS solution was prepared using a 1:5 ratio of MTS reagent and D-MEM 


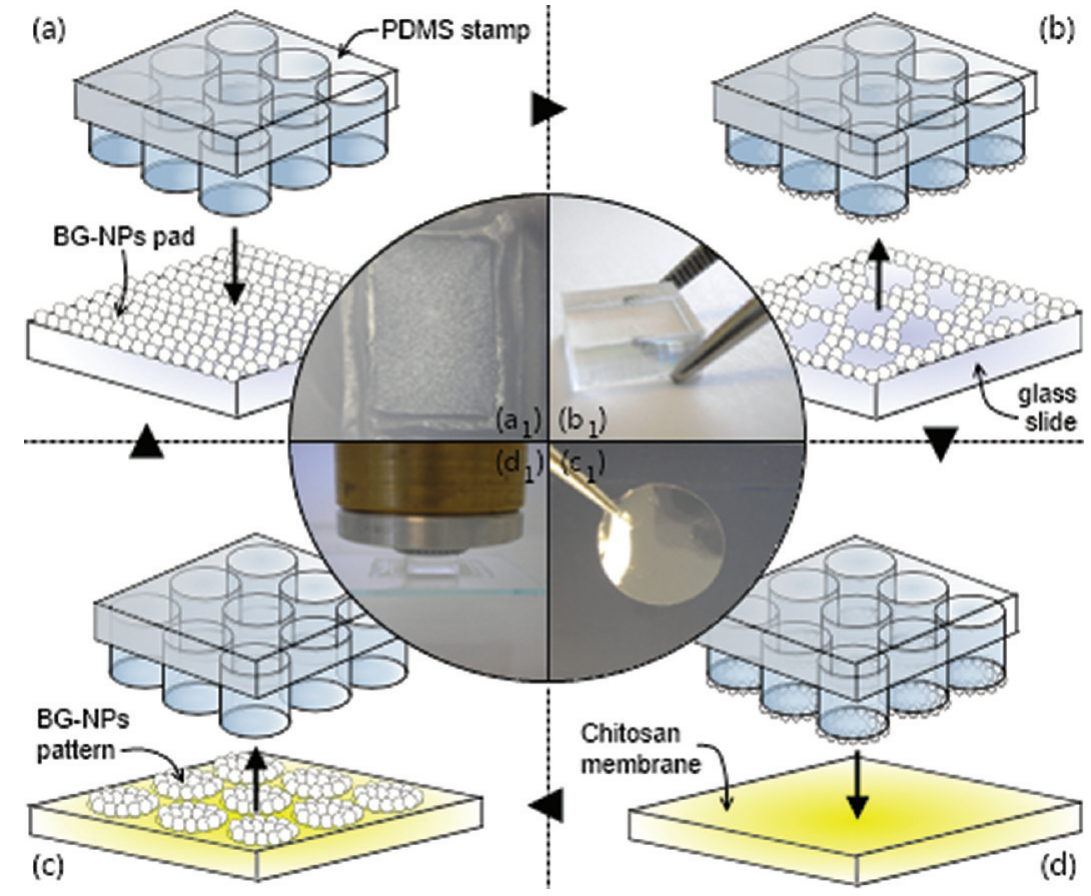

Figure 1. Schematic illustration and photographs of the materials used and the procedure followed: (a) Inking of the PDMS stamp in a glass substrate covered with a homogeneous layer of BG-NPs (a1); (b) Lift-off of the PDMS stamp carrying the BG-NPs on the base of the features and PDMS stamp (b1); (c) Pressing of the PDMS stamp in the chitosan membrane's surface, and chitosan membrane used as printing substrate (c1); (d) Printing of BG-NPs over the chitosan membrane's surface, and detail of the device used to press the stamp against the substrate (d1). Same stamp may be utilized again after washing with ethanol in such imprinting procedure.

culture medium without phenol red or FBS, followed by a $3 \mathrm{~h}$ incubation period at $37^{\circ} \mathrm{C}$. All cytotoxicity tests were conducted using 3 replicates. Finally, the optical density (OD) was read at $490 \mathrm{~nm}$ on a multiwell microplate reader (Synergy HT, Bio-Tek Instruments).

At days 1, 3, and 7 after seeding, cell attachment on the membranes was followed by SEM. Membranes were rinsed twice with PBS to remove nonadherent or loosely adherent cells and fixed with a solution of $2.5 \%(\mathrm{v} / \mathrm{v})$ glutaraldehyde in $0.1 \mathrm{M}$ PBS for $1 \mathrm{~h}$ at $4{ }^{\circ} \mathrm{C}$. After removing the fixative, cells were rinsed in PBS and distilled water and dehydrated in a graded series of ethanol solution $(50 \%, 70 \%, 90 \%$, and $100 \%)$, each one repeated twice for $15 \mathrm{~min}$. They were left to dry at air at room temperature and sputter coated with gold before SEM observation.

For the fluorescent images, calcein AM was used to stain the cells. A $2 \mu \mathrm{L}$ amount of calcein AM and $1 \mathrm{~mL}$ of D-MEM culture medium without phenol red or FBS were added to each sample, followed by 10 min of incubation at $37^{\circ} \mathrm{C}$. Live cells stained green due to enzymatic conversion of the nonfluorescent cell-permeant calcein AM to fluorescent calcein. Fluorescent cells were visualized with the corresponding filters under an inverted microscope (Imager-Z1M).

\section{RESULTS AND DISCUSSION}

The procedure employed to load the BG-NPs onto chitosan membranes has been described as a "stamp pad"33 or "stickand-place" ${ }^{34}$ method. The PDMS stamp was placed in contact with a glass slide coated with the BG-NPs (donor substrate). The stamp picked up the nanoparticles, and these were printed onto the chitosan membrane (receiving substrate). Figure 1 represents the different steps of this process. Ethanol was used to produce the BG-NPs ink as it is known to have a minimal swelling effect on the PDMS stamp. ${ }^{35}$ PDMS was the selected stamp material since it has enough rigidity to support the topographic microstructure while it is soft and bendable, allowing conformal contact between the stamp and the membrane. ${ }^{11,33,36,37}$
Different processing conditions were tested in the production of the patterned membranes, namely, the pattern shape and features height, the BG-NPs "ink" concentration, the pressure applied, and the contact time. Although $\mu \mathrm{CP}$ is usually performed manually, in this work a homemade transfer device was used in order to have accurate control of the pressure applied to the stamp/substrate interface-see Figure 2.

3.1. Pattern Formation. Three geometries of PDMS stamps were used in this study: $50 \mu \mathrm{m}$ diameter cylindrical

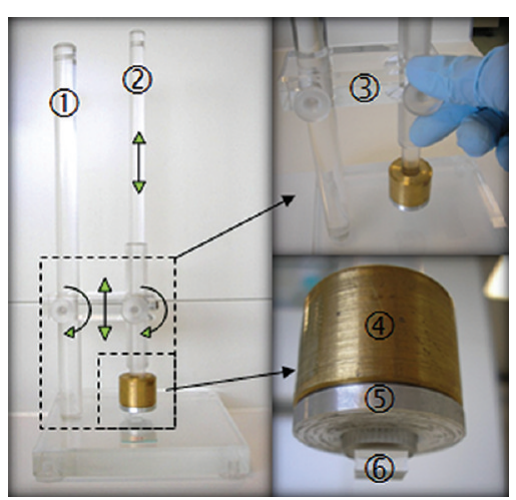

Figure 2. Device is mainly composed by two axes. One of the axes (1) is fixed to the base of the device and will be the guide of the central piece (3). This central piece will establish the connection between the fixed axe and the movable axe where the weights are applied. Fixed axe provides stability during the printing process. There are two screws in piece 3. One will fix the central piece to the fixed axe, and the other will fix or loosen the position of the movable axe. Different weights (4) have a hole in the middle in order to fit in the movable axe. Weight of the axe plus the gray disk (5) is exactly $50 \mathrm{~g}$. PDMS stamp (6) is glued to the bottom of the movable axe with a double-sided adhesive tape. 

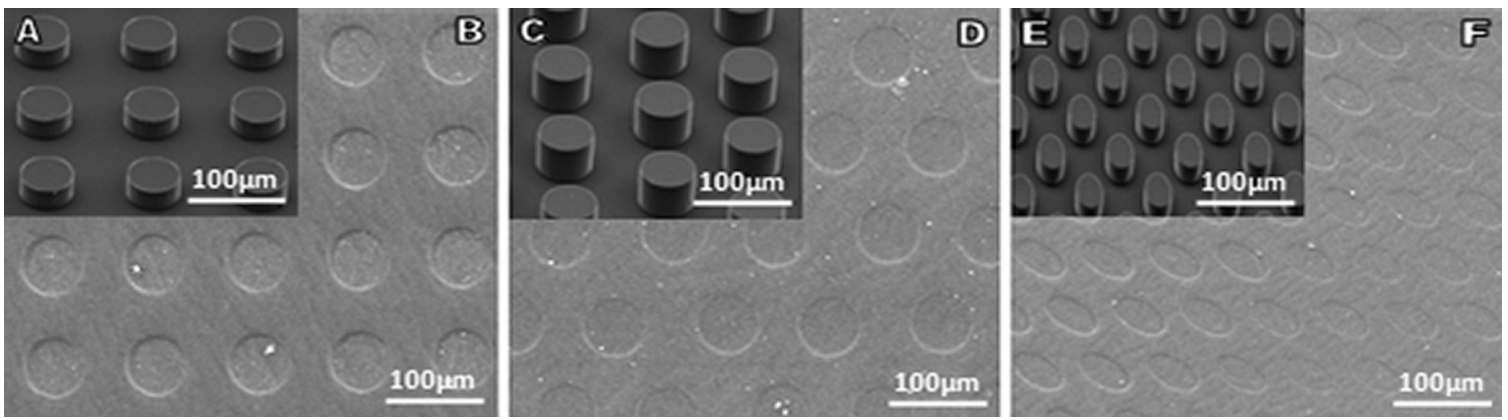

Figure 3. SEM images of types of patterns used: (a) PDMS stamp and (b) chitosan membrane patterned with $50 \mu \mathrm{m}$ diameter circles aligned in a squared arrangement; (c) PDMS stamp and (d) chitosan membrane patterned with $50 \mu$ m diameter circles forming a hexagonal pattern; (e) PDMS stamp and (f) chitosan membrane patterned with ellipses aligned in a hexagonal arrangement with the higher axis reaching $50 \mu \mathrm{m}$.

pillars arranged in a square pattern with $50 \mu \mathrm{m}$ spacing (Figure 3a), $50 \mu \mathrm{m}$ diameter cylindical pillars arranged in a hexagonal pattern with $40 \mu \mathrm{m}$ spacing (Figure $3 \mathrm{c}$ ), and ellipsoidal pillars (50 $\mu \mathrm{m}$ long axes and $30 \mu \mathrm{m}$ short axes) arranged in a hexagonal pattern with $40 \mu \mathrm{m}$ spacing (Figure 3e). SEM images of the corresponding BG-NPs patterns obtained after stamping on the chitosan membranes are shown in Figure 3b, 3d, and $3 f$. The diameter and spacing of the features needed to be optimized for our particular application and materials used. Stamps with features with 20,30 , and $40 \mu \mathrm{m}$ height were tested for stamping. The pattern formed by the PDMS stamp with 30 $\mu \mathrm{m}$ height features seemed to combine the nondeformability with adequate BG-NPs transfer from the stamp to the membrane and gave the best results using a BG-NPs concentration of $0.03 \%(\mathrm{w} / \mathrm{v})$. Well-defined and nondeformed circles of homogeneously distributed BG-NPs were obtained on the membranes. Another reason for choosing this pattern design was to have enough area available within the patterned fields to allow cell proliferation but also to have enough space between the features to allow good resolution of the pattern and avoid contamination of BG-NPS outside of the desired areas.

The force applied to the stamp during contact with the membrane influences pattern formation and reproducibility. ${ }^{11}$ Due to the elastomeric and soft nature of the PDMS, excess applied vertical pressure during printing causes distortion of the patterns. ${ }^{11,37}$ Reproducibility could be greatly improved using a mechanical device that allowed accurate control of the applied pressure. The effect of the contact time between the PDMS stamp and the BG-NPs membrane substrate was also studied. No important differences in the obtained patterns were found for contact times between $30 \mathrm{~s}$ and $10 \mathrm{~min}$. This result agrees with previously reported data. ${ }^{12}$ When printing the BG-NPs in the chitosan membrane, acetic acid was used to treat the membrane and promote covalent binding between the polymer and the BG-NPs, since, otherwise, BG-NPs would remain attached to the PDMS stamp rather than transfer to the chitosan membrane. Both surface chemistries of the stamp and substrate are important in determining transfer efficiency. ${ }^{37}$ For the particles to transfer onto the substrate, binding to the new surface must be more energetically favorable than remaining on the stamp. The process of transferring between stamp/ink is modeled with a speed-dependent critical energy release rate, which increases with speed due to the viscoelastic nature of PDMS. $^{34}$ Thus, fast peeling rates are used initially to transfer objects from a surface to PDMS, and slow rates are used to transfer these objects to a new unpatterned surface. ${ }^{36}$ In addition, temperature is also an important parameter to consider in patterning systems. However, the rate of stamp removal and the temperature during the printing experiment were kept constant for all experiments.

3.2. Mineralization Studies. Mineralization of the BGNPs patterns was assessed in vitro by analyzing the ability of the patterned membrane to induce precipitation of apatite upon immersion in SBF. The patterned membrane before immersion in SBF showed small clusters of BG-NPs randomly distributed although well confined in the circular stamped regions-see Figure 4a. BG-NPs tend to agglomerate in clusters of variable sizes. However, individually, these particles present rounded shape morphology with sizes around $40 \mathrm{~nm} .{ }^{30}$ After immersing the entire membrane in SBF for 5 days, well-defined
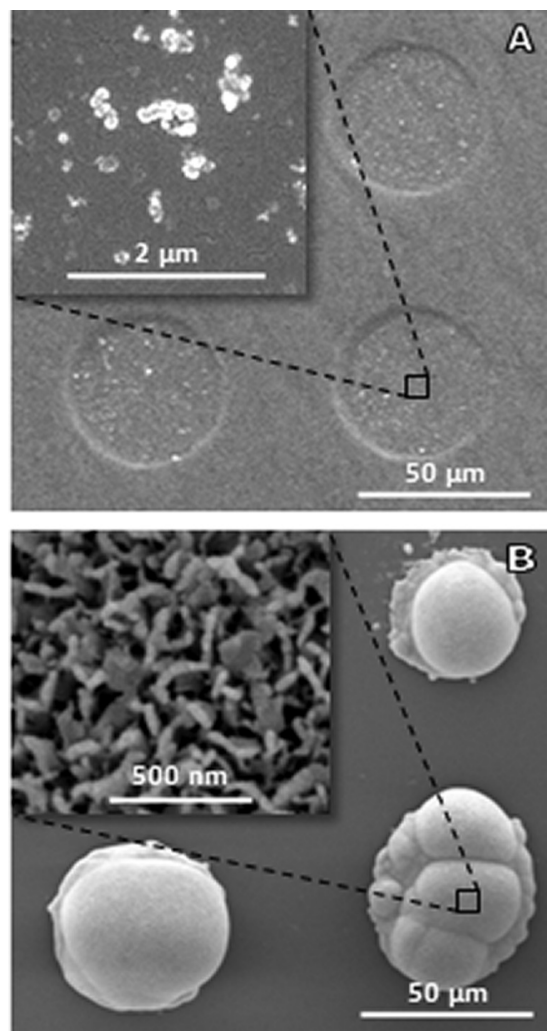

Figure 4. (a) SEM images of BG-NPs pattern on chitosan before immersion in SBF. (b) SEM images of the patterned chitosan membrane evidencing the calcified clusters after 5 days of soaking in SBF. 
cauliflower-like clusters were detected. These were formed by platelets similar to the ones found in the typical structures of biomimetically formed apatite-see Figure $4 \mathrm{~b}$. Mineralization occurred only in the patterned areas where BG-NPs were present, proving the confinement of controlled mineralized patterns.

EDX analyses of the membrane were performed in order to analyze the change in the surface chemical composition related to the mineralization process-see Figure 5. A slight increase in

(a)

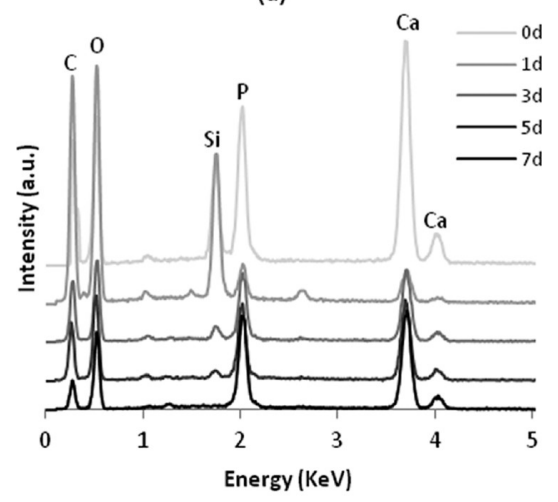

(b)

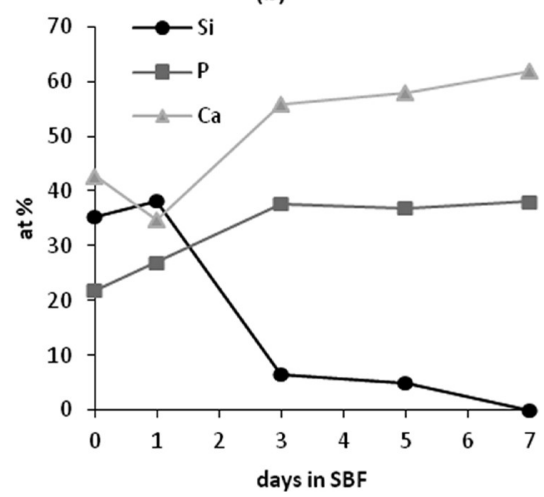

Figure 5. (a) EDX spectra and (b) relative compositions of Si, $\mathrm{P}$, and $\mathrm{C}$ are given (in atomic percent, atom \%) from EDX analysis upon the mineralization study of the BG-NPS-patterned chitosan membranes soaked in SBF for different time points (0 (control), 1, 3, 5, and 7 days).

the fraction of Si in the membrane and a decrease of Ca until day 1 was observed. This is attributed to the exchange of $\mathrm{Ca}$ ions from the sample and $\mathrm{H}^{+}$and $\mathrm{H}_{3} \mathrm{O}^{+}$from the $\mathrm{SBF}$ solution. After day 1, disruption of the glass network occurs by hydrolysis and soluble silica is lost to the solution in the form of $\mathrm{Si}(\mathrm{OH})_{4}$, which leads to formation of silanol groups $(\mathrm{Si}-\mathrm{OH})$ at the $\mathrm{BG}-\mathrm{NPs} /$ solution interface. The $\mathrm{OH}^{-}$groups from silanol attract $\mathrm{Ca}$ from the surrounding media, and precipitation occurs. An amorphous calcium phosphate layer begins to grow on the surface of the sample, as can be confirmed by the $\mathrm{Ca}$ and $\mathrm{P}$ increasing values. In contrast, $\mathrm{Si}$ content decreases dramatically. Incorporation of $\mathrm{OH}^{-}$and $\mathrm{CO}_{3}{ }^{2-}$ anions from solution until day seven leads to the final step of mineralization which is formation of a crystalline apatite layer. $^{38}$

The value of the $\mathrm{Ca} / \mathrm{P}$ ratio of the nonstochiometric apatitic layer developed on the surface of the BG-NPs was 1.63 in day 7. This value is very close to the one attributed to stoichiometric hydroxyapatite, $1.67,{ }^{39}$ implying that a hydroxyapatite layer resembling the natural bone mineral phase is being formed at the end of 7 days of immersion in SBF.

Evolution of apatite formation was also analyzed using FTIR-see Figure 6. The spectra presented correspond to the

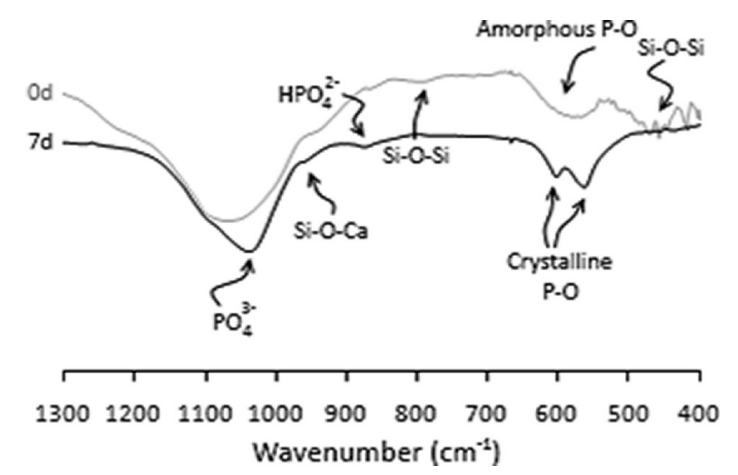

Figure 6. FTIR spectra of the powders scratched from the surface of the patterned membranes after 0 (control) and 7 days of immersion in SBF.

apatite powder scratched from the patterned membranes after 0 and 7 days of immersion in SBF. Silicate absorption bands are observed in all spectra, assigned to the bands 1085, 800, and $464 \mathrm{~cm}^{-1}$, respectively: asymmetric stretching mode, symmetric stretching vibration, and rocking vibration of $\mathrm{Si}-\mathrm{O}-\mathrm{Si}^{40}$ Silicate-related bands are more intense in the control sample, which is in accordance with previous observations that the Si content decreases after being soaked in SBF.

Major evidence of hydroxyapatite growth is based on the bands at 600 and $550 \mathrm{~cm}^{-1}$ related to the $\mathrm{P}-\mathrm{O}$ bending vibration due to the presence of a crystalline calcium phosphate (apatite like) phase. After 7 days in SBF the amorphous band in the control sample at around $600 \mathrm{~cm}^{-1}$ in SBF evolves to these two bands, evidencing the phosphate groups in the hydroxyapatite crystalline conformation. The bands at 1045 and $1090 \mathrm{~cm}^{-1}$ are also assigned to the $\mathrm{P}-\mathrm{O}$ bond, and they increase in the 7 days sample. ${ }^{41}$ The band at $874 \mathrm{~cm}^{-1}$ is assigned to the acidic phosphate group $\left(\mathrm{HPO}_{4}{ }^{2-}\right){ }^{42}$ The presence of $\mathrm{Ca}$ is assigned by the band at $950 \mathrm{~cm}^{-1}$ related to $\mathrm{Si}-\mathrm{O}-\mathrm{Ca}$ bonds containing nonbridging oxygen in opposition with the bridging oxygens that link two $\mathrm{SiO}_{4}$ tetrahedra. ${ }^{40,43}$

Soaking of the BG-NPs in SBF for 7 days was already proved to result in a crystalline apatitic layer. ${ }^{30}$ In a previous study performed by the authors, XRD data show that the amorphous spectra of the BG-NPs, after immersion in SBF for 7 days, resulted in peaks with diffraction angles matching the reference X-ray spectra of hydroxyapatite.

3.3. Cellular Viability. The cytotoxicity of the patterned chitosan membranes was assessed using a direct contact MTS assay-see Figure 7. All results for both patterned and unpatterned (pure chitosan) membranes of relative viability (\%) of the L929 cells were normalized to cell viability on TCPS, which was used as a positive control of cell viability. Latex substrates were used as a negative control for cellular viability, and after 7 days of culture the percentage of cell viability in latex was considered to be negligible $(<0.5 \%)$. The results revealed that the cells seeded on the BG-NPs-patterned membranes exhibited increased cell viability over the culture time points (1, 3, and 7 days). Moreover, the viability of cells seeded on patterned membranes was considerably superior to 


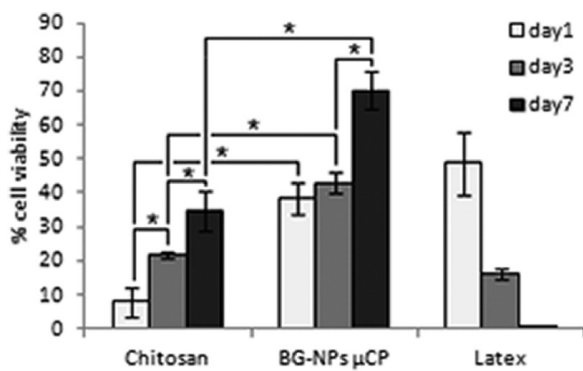

Figure 7. Cell viability of the produced samples from MTS tests throughout 7 days of culture as compared with the cells cultured in TCPS. Data are means \pm SD $(n=3 ; *=p<0.05)$.

that observed in plain chitosan membranes. The results obtained in the MTS tests prove the important role of the BG-NPs in promoting cellular viability and are also supported by the morphological observation study.

In order to study cellular morphology and position at the surfaces, L929 cells were seeded on the control (plain chitosan membranes) and BG-NPs-patterned membranes at a density of $60000 \mathrm{cell} / \mathrm{cm}^{2}$. Figure 8 presents SEM and fluorescence microscopy images of the membranes for different culture times: 1, 3, and 7 days. In the patterned membranes cells adhered and proliferated mainly in the areas printed with BGNPs. In addition, the density of cells on the patterned substrate increased with increasing culture time, always respecting the printed BG-NPs pattern. The BG-NPs-confined area proved to be a highly bioreactive surface which can promote attachment of living cells. Although cells present a flattened morphology, the control membranes of plain chitosan did not favor proliferation of cells throughout the time study, compared to the patterned membranes, consistent with the MTS results. Cells cultured on the patterned BG-NPs membranes appear to be relatively well spread and highly flattened with an increased cell-substrate contact area ratio. Formation of pseudopodia was also observed. However, at day 7 , cells appear to exhibit a relatively rounded unspread morphology, probably due only to the lack of space since the MTS results proved their viability. In this case cells preferred to adopt a more dense geometry and form stacked arrangements over the regions containing BGNPs rather than migrate and attach to pure chitosan regions. The cell stack observed in Figure $8 \mathrm{~g}$ indicates that the higher located cells, not able to sense neither the nano- nor the microtopography, prefer the environment created by the BGNPs ionic release rather than migrating to chitosan.

\section{CONCLUSIONS}

For the first time $\mu \mathrm{CP}$ has been used to pattern mineralizable elements onto a biomaterial. Chitosan membranes were used and circular motifs containing bioactive glass nanoparticles were printed on them using previously inked PDMS stamps. The bioactive character of the BG-NPs spots allowed nucleation and growth of apatite, highly localized in the patterned regions of the chitosan membranes. By combining the remarkable properties of BG-NPs and the excellent biocompatibility of chitosan with this simple microcontact printing approach it was proved that it is possible to control the
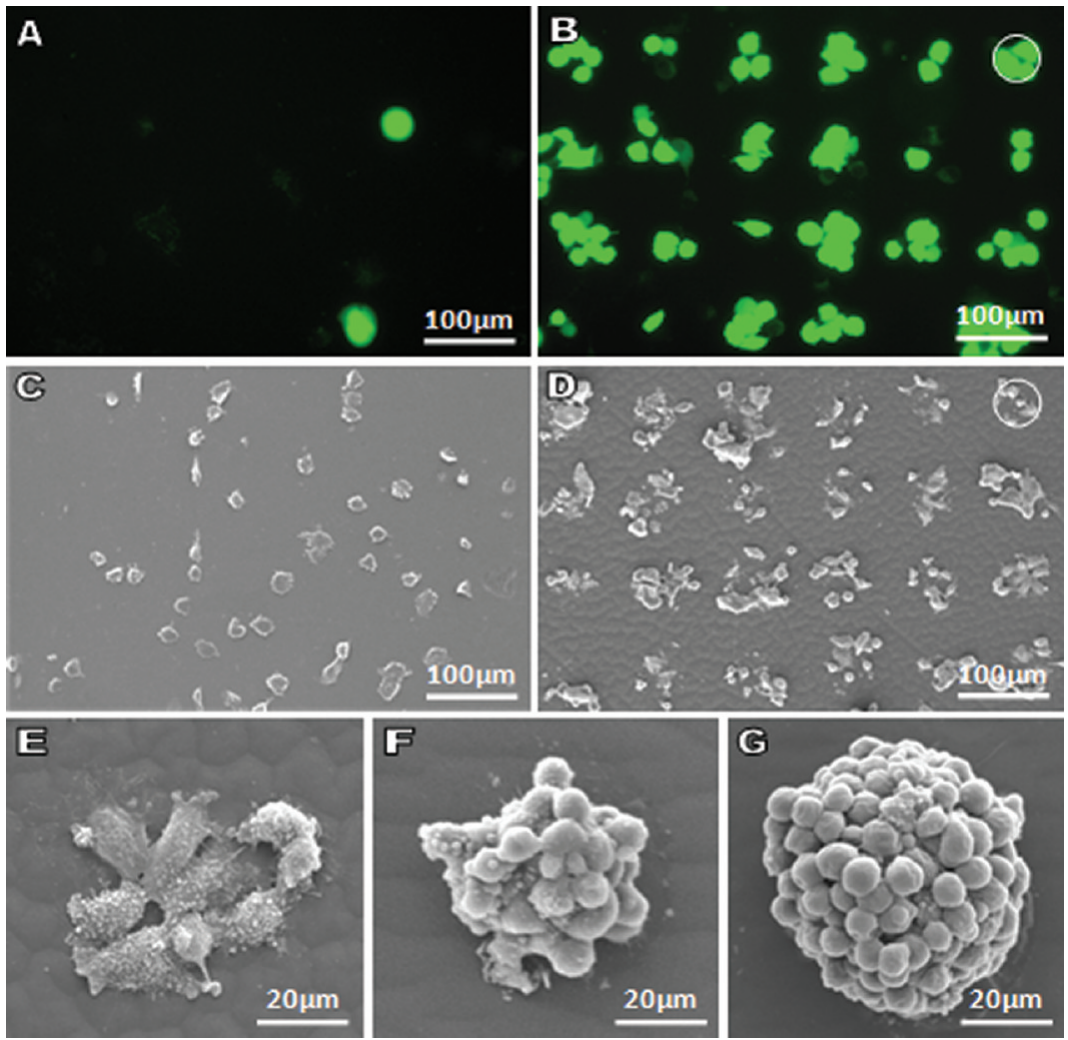

Figure 8. Fluorescent images of cell stained with calcein AM after 1 day of culture in plain chitosan (a) and BG-NPs patterned membranes (b); SEM images of 1 day of cell culture in plain chitosan (c) and BG-NPs patterned membranes (d); SEM images magnification of $24 \mathrm{~h}$ (e), 3 days (f), and 7 days $(\mathrm{g})$ of cell culture in BG-NPs patterned membranes; circle of $50 \mu \mathrm{m}$ of diameter was inserted in the top (right) of images $\mathrm{b}$ and $\mathrm{d}$ for indication purposes. 
cellular interactions with a bioactive substrate at the microscale level. Successful patterning of fibroblasts is an indication of the versatility of the developed system.

This technology can be extended to studies of patterns with different sizes, geometries, and orientations that are known to influence cell differentiation and also develop cellular cocultures. The approach proposed opens also new routes of developing patterned medical membranes with distinct properties in the two sides, able to promote guided tissue regeneration in the bone side, while preventing soft tissue growth on the other face.

\section{AUTHOR INFORMATION}

\section{Corresponding Author}

*E-mail: jmano@dep.uminho.pt.

\section{Notes}

The authors declare no competing financial interest.

\section{ACKNOWLEDGMENTS}

This work was supported by the Portuguese Foundation for Science and Technology (FCT), through project PTDC/ CTM-BPC/112774/2009 and the $\mathrm{PhD}$ grant SFRH/BD/ 45777/2008. A.d.C. acknowledges financial support from the Max-Planck-Gesellschaft and Fraunhofer joint project "Biomimetische Matrices”.

\section{REFERENCES}

(1) Hench, L. L.; Thompson, I. Twenty-first century challenges for biomaterials. J. R. Soc. Interface 2010, 7, S379-S391.

(2) Hench, L. L.; Polak, J. M. Third-generation biomedical materials. Science 2002, 295 (5557), 1014.

(3) Alves, N. M.; et al. Controlling Cell Behavior Through the Design of Polymer Surfaces. Small 2010, 6 (20), 2208-2220.

(4) McBeath, R; et al. Cell shape, cytoskeletal tension, and RhoA regulate stem cell lineage commitment. Dev. Cell 2004, 6 (4), 483495.

(5) Boyan, B. D.; et al. Osteoblast-mediated mineral deposition in culture is dependent on surface microtopography. Calcified Tissue Int. 2002, 71 (6), 519-529.

(6) Curtis, A.; Wilkinson, C. Topographical control of cells. Biomaterials 1997, 18 (24), 1573-1583.

(7) Kumar, G.; et al. Spatially controlled cell engineering on biomaterials using polyelectrolytes. Langmuir 2003, 19 (25), 1055010556.

(8) Park, T. H.; Shuler, M. L. Integration of cell culture and microfabrication technology. Biotechnol. Prog. 2003, 19 (2), 243-253.

(9) Wilbur, J. L.; et al. Microfabrication by microcontact printing of self-assembled monolayers. Adv. Mater. 1994, 6 (7-8), 600-604.

(10) Corum, L. E.; et al. Using Microcontact Printing of Fibrinogen to Control Surface-Induced Platelet Adhesion and Activation. Langmuir 2011, 27 (13), 8316-8322.

(11) Quist, A. P.; Pavlovic, E.; Oscarsson, S. Recent advances in microcontact printing. Anal. Bioanal. Chem. 2005, 381 (3), 591-600.

(12) Andersson, A. S.; et al. Patterning colloidal monolayer films using microcontact particle stripping. Nanotechnology 2007, 18, 20.

(13) Yan, X.; et al. Microcontact printing of colloidal crystals. J. Am. Chem. Soc. 2004, 126 (34), 10510-10511.

(14) Xia, Y. N.; Whitesides, G. M. Soft lithography. Annu. Rev. Mater. Sci. 1998, 28, 153-184.

(15) Torisawa, Y.-S.; et al. Transwells with Microstamped Membranes Produce Micropatterned Two-Dimensional and ThreeDimensional Co-Cultures. Tissue Eng., Part C: Methods 2011, 17 (1), 61-67.

(16) Hench, L. L.; Polak, J. M. Third-generation biomedical materials. Science 2002, 295, 1014-1017.
(17) Alves, N. M.; et al. Designing biomaterials based on biomineralization of bone. J. Mater. Chem. 2010, 20 (15), 2911-2921.

(18) Hong, Z.; et al. Mono-dispersed bioactive glass nanospheres: Preparation and effects on biomechanics of mammalian cells. J. Biomed. Mater. Res. A 2010, 95A (3), 747-754.

(19) Ozawa, N.; Yao, T. Micropattern formation of apatite by combination of a biomimetic process and transcription of resist pattern. J. Biomed. Mater. Res. 2002, 62 (4), 579-586.

(20) Shi, J.; Alves, N. M.; Mano, J. F. Thermally responsive biomineralization on biodegradable substrates. Adv. Funct. Mater. 2007, 17 (16), 3312-3318.

(21) Hench, L. L.; Polak, J. M. Third-generation biomedical materials. Science 2002, 295, 1014-1017.

(22) Boccaccini, A. R.; et al. Polymer/bioactive glass nanocomposites for biomedical applications: A review. Compos. Sci. Technol. 2010, 70 (13), 1764-1776.

(23) Luz, G. M.; Mano, J. F. Mineralized structures in nature: Examples and inspirations for the design of new composite materials and biomaterials. Compos. Sci. Technol. 2010, 70 (13), 1777-1788.

(24) Labbaf, S.; et al. Spherical bioactive glass particles and their interaction with human mesenchymal stem cells in vitro. Biomaterials 2011, 32 (4), 1010-8.

(25) Mackay, C. E.; et al. Stochastic probability modeling to predict the environmental stability of nanoparticles in aqueous suspension. Integr. Environ. Assess. Manage. 2006, 2 (3), 293-8.

(26) Schaefer, S.; et al. How Degradation of Calcium Phosphate Bone Substitute Materials is influenced by Phase Composition and Porosity. Adv. Eng. Mater. 2011, 13 (4), 342-350.

(27) Misra, S. K.; et al. Comparison of nanoscale and microscale bioactive glass on the properties of $\mathrm{P}(3 \mathrm{HB}) /$ Bioglass $(\mathrm{R})$ composites. Biomaterials 2008, 29 (12), 1750-1761.

(28) Auffan, M.; et al. Towards a definition of inorganic nanoparticles from an environmental, health and safety perspective. Nat. Nanotechnol. 2009, 4 (10), 634-641.

(29) Hong, Z. K.; Reis, R. L.; Mano, J. F. Preparation and in vitro characterization of scaffolds of poly(L-lactic acid) containing bioactive glass ceramic nanoparticles. Acta Biomaterialia 2008, 4 (5), 12971306.

(30) Luz, G.; Mano, J. F. Preparation and characterization of bioactive glass nanoparticles prepared by sol-gel for biomedical applications. Nanotechnology, 2011. 22.

(31) Greiner, C.; del Campo, A.; Arzt, E. Adhesion of bioinspired micropatterned surfaces: Effects of pillar radius, aspect ratio, and preload. Langmuir 2007, 23 (7), 3495-3502.

(32) Kokubo, T.; Takadama, H. How useful is SBF in predicting in vivo bone bioactivity? Biomaterials 2006, 27 (15), 2907-2915.

(33) Pompe, T.; et al. Submicron contact printing on silicon using stamp pads. Langmuir 1999, 15 (7), 2398-2401.

(34) Feng, X.; et al. Competing fracture in kinetically controlled transfer printing. Langmuir 2007, 23, 12555-12560.

(35) Perl, A.; Reinhoudt, D. N.; Huskens, J. Microcontact Printing: Limitations and Achievements. Adv. Mater. 2009, 21 (22), 2257-2268.

(36) Bowden, N. Micromanipulation: Stick and place. Nat. Mater. 2006, 5 (1), 9-10.

(37) Ruiz, S. A.; Chen, C. S. Microcontact printing: A tool to pattern. Soft Matter 2007, 3 (2), 168-177.

(38) Zhong, J. P.; Greenspan, D. C. Processing and properties of solgel bioactive glasses. J. Biomed. Mater. Res. 2000, 53 (6), 694-701.

(39) Koutsopoulos, S. Kinetic study on the crystal growth of hydroxyapatite. Langmuir 2001, 17 (26), 8092-8097.

(40) Ma, J.; et al. In vitro degradability and bioactivity of mesoporous $\mathrm{CaO}-\mathrm{MgO}-\mathrm{P} 2 \mathrm{O} 5-\mathrm{SiO} 2$ glasses synthesized by sol-gel method. J. SolGel Sci. Technol. 2010, 54 (1), 69-76.

(41) Hong, Z.; Reis, R. L.; Mano, J. F. Preparation and in vitro characterization of novel bioactive glass ceramic nanoparticles. $J$. Biomed. Mater. Res., Part A 2009, 88A (2), 304-313.

(42) Zhang, H. Q.; et al. Precipitation of biocompatible hydroxyapatite whiskers from moderately acid solution. Ceram. Int. 2003, 29 (4), 413-418. 
(43) Stebbins, J. F.; Xu, Z. NMR evidence for excess non-bridging oxygen in an aluminosilicate glass. Nature 1997, 390 (6655), 60-62. 